Virginia Commonwealth University VCU Scholars Compass

2007

\title{
Structure of $\mathrm{SiAu}(16)$ : Can a silicon atom be stabilized in a gold cage?
}

Qiang Sun

Peking University, Virginia Commonwealth University, qsun@vcu.edu

Qian Wang

Virginia Commonwealth University

Gang Chen

Virginia Commonwealth University

Puru Jena

Virginia Commonwealth University, pjena@vcu.edu

Follow this and additional works at: http://scholarscompass.vcu.edu/phys_pubs

Part of the Physics Commons

Sun, Q. Wang, Q. Chen, G., et al. Structure of Si Au 16 : Can a silicon atom be stabilized in a gold cage?. The Journal of Chemical Physics 127, 214706 (2007). Copyright @ 2007 AIP Publishing LLC.

\section{Downloaded from}

http://scholarscompass.vcu.edu/phys_pubs/189

This Article is brought to you for free and open access by the Dept. of Physics at VCU Scholars Compass. It has been accepted for inclusion in Physics Publications by an authorized administrator of VCU Scholars Compass. For more information, please contact libcompass@vcu.edu. 


\title{
Structure of $\mathrm{SiAu}_{16}$ : Can a silicon atom be stabilized in a gold cage?
}

\author{
Qiang Sun ${ }^{\text {a) }}$ \\ Department of Advanced Materials and Nanotechnology, Peking University, Beijing 100871, China \\ and Physics Department, Virginia Commonwealth University, Richmond, Virginia 23284, USA
}

Qian Wang, Gang Chen, and Puru Jena

Physics Department, Virginia Commonwealth University, Richmond, Virginia 23284, USA

(Received 5 July 2007; accepted 5 October 2007; published online 7 December 2007)

\begin{abstract}
Nanostructures of $\mathrm{Au}$ and $\mathrm{Si}$ as well as $\mathrm{Au}-\mathrm{Si}$ hybrid structures are topics of great current interest from both scientific and technological points of view. Recent discovery of Au clusters having fullerenelike geometries and the possibility of endohedral complexes with $\mathrm{Si}$ atoms inside the $\mathrm{Au}$ cage opens new possibilities for designing $\mathrm{Au}-\mathrm{Si}$ nanostructures. Using ab initio simulated annealing method we have examined the stability of $\mathrm{Si}-\mathrm{Au}_{16}$ endohedral complex. Contrary to what we believed, we find that the endohedral configuration is metastable and the structure where $\mathrm{Si}$ atom binds to the exterior surface of the $\mathrm{Au}_{16}$ cage is the lowest energy structure. The bonding of $\mathrm{Si}$ to $\mathrm{Au}$ cluster mimics its behavior of that in bulk and liquid phase of Au. In addition, doping of Si in high concentration would cause fracture and embrittlement in gold nanostructures just as it does in the bulk phase. Covalent bonding between $\mathrm{Au}-\mathrm{Au}$ and $\mathrm{Au}-\mathrm{Si}$ is found to be a dominant feature in the stability of the Au-Si nanostructures. Our study provides insight that may be useful in fabricating hybrid $\mathrm{Au}-\mathrm{Si}$ nanostructures for applications microelectronics, catalysis, biomedine, and jewelry industry. (C) 2007 American Institute of Physics. [DOI: 10.1063/1.2804872]
\end{abstract}

Gold and silicon are two of the most important elements in the Periodic Table. Gold is the noblest of all metals and is prized throughout history for its beauty and resistance to corrosion. ${ }^{1}$ Silicon, on the other hand, forms the basis for electronics. The interaction of $\mathrm{Si}$ and $\mathrm{Au}$ exhibits some unique features: Although $\mathrm{Au}$ and $\mathrm{Si}$ do not form any stable crystalline alloys at any concentration and temperature, $\mathrm{SiAu}_{4}$ (Ref. 2) commonly known as aurosilane is a very stable structure where four of the Au atoms behave just like hydrogen atoms. This raises the following question: Can $\mathrm{Si}$ be incorporated into nano gold? This is particularly important as novel nanoelectronic devices can be envisioned by creating Au-Si interface. In addition, the discovery ${ }^{3}$ of reactive gold nanoparticles has caused a great deal of interest in exploring the synthesis of gold at the nanoscale. ${ }^{4}$ It was recently demonstrated ${ }^{5}$ that the $\mathrm{Au}_{16}$ cluster can form a cage structure similar to that of carbon fullerenes. The possibility of having pure metal cage that can be functionalized with endohedral atoms opens a new area research with potential for technological breakthroughs. For example, Au can be used for catalysis ${ }^{6}$ and can be easily functionalized for biomedical applications in drug delivery, hyperthermal treatment, and magnetic separation. ${ }^{7-9}$

It was recently suggested that $\mathrm{Au}_{16}$ which has a cage structure with a distorted $T_{d}$ geometry can be endohedrally doped with $\mathrm{Si}$ making a $\mathrm{Si}-\mathrm{Au}_{16}$ cluster. ${ }^{10}$ Such cluster, analogous to $\mathrm{Si}-\mathrm{Al}_{12}$ (Ref. 11) can have 20 delocalized electrons and mimic a magic cluster due to electronic shell closure. The study of $\mathrm{Si}-\mathrm{Au}_{16}$ raises the following interesting

\footnotetext{
a) Author to whom correspondence should be addressed. Electronic mails: sunq@coe.pku.edu.cn and qsun@vcu.edu
}

question: Does the interaction of $\mathrm{Si}$ with $\mathrm{Au}_{16}$ resemble that in aurosilane or bulk phase? Recall that the former is a very stable species while the later does not form any stable crystalline alloy. The predicted stability ${ }^{10}$ of endohedral $\mathrm{Si}-\mathrm{Au}_{16}$ cluster would suggest that the interaction is dominated by the $s p^{3}$ bonding characteristics of $\mathrm{Si}$ and a nanoalloy of $\mathrm{Si}-\mathrm{Au}$ is possible even though its bulk counter part does not exist.

We have examined the stability of endohedral $\mathrm{Si}-\mathrm{Au}_{16}$ by carrying out $a b$ initio simulated annealing calculation with different starting geometries. We show that the endohedral doping belongs to a metastable configuration. The lowest energy structure is that of the $\mathrm{Si}$ atom bonding on the surface site of the $\mathrm{Au}_{16}$ cluster and is $0.457 \mathrm{eV}$ lower in energy than the metastable endohedral complex. Thus, the outer surface of nanogold structure is more reactive for $\mathrm{Si}$ doping than its interior, similar to that of gold bulk. The results indicate that the bonding between $\mathrm{Si}$ and gold is not the same as that between $\mathrm{Si}$ and $\mathrm{Al}$ and that the electronic shell closure may not be the leading contributor to the stability of the $\mathrm{Si}-\mathrm{Au}_{16}$ complex.

Our calculations are based on spin-polarized density functional theory with generalized gradient approximation (GGA) for exchange and correlation potentials. We have used the Perdew-Burke-Ernzerhof form for the GGA and a plane-wave basis set with the projector augmented plane wave method as implemented in the Vienna $a b$ initio simulation package (VASP). ${ }^{12,13}$ Supercells with $15 \AA$ vacuum spaces along the $x, y$, and $z$ directions for all the calculated structures are used. Due to the large supercell the $\Gamma$ point is used to represent the Brillouin zone. The geometries of the structures are optimized without symmetry constraint. The energy cutoff was set to $300 \mathrm{eV}$ and the convergence in en- 


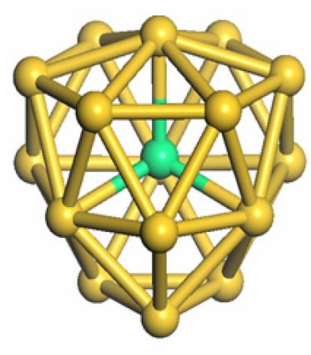

$\mathrm{DE}=0.0 \mathrm{eV}$ gap $=1.875 \mathrm{eV}$

(a)

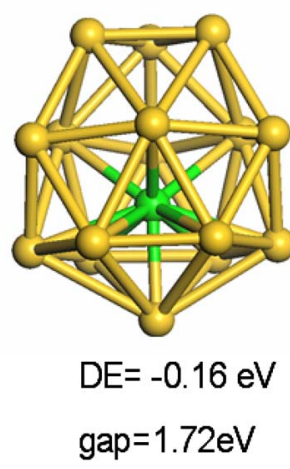

(b)

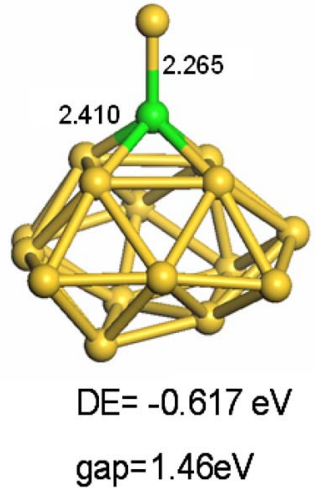

(c) ergy and force were $10^{-4} \mathrm{eV}$ and $1 \times 10^{-3} \mathrm{eV} / \AA$, respectively. The accuracy of our numerical procedure was tested for $\mathrm{Au}_{2}$ and $\mathrm{AuSi}$ dimers. The calculated bond lengths for $\mathrm{Au}_{2}$ and $\mathrm{AuSi}$ are 2.526 and $2.251 \AA$, respectively, which agree very well with corresponding experimental values ${ }^{14,15}$ of 2.500 and $2.257 \AA$. For larger gold nanostructures, readers can refer to our previous studies. ${ }^{16}$ Tests were also carried out for $\mathrm{Si}-\mathrm{Au}_{16}$ with on-center and off-center configurations, as shown in Figs. 1(a) and 1(b), the latter is found to be $0.16 \mathrm{eV}$ lower in energy, in agreement with the results in Ref. 10. Although these two isomers have nearly the same energy, frequency calculations indicate that the on-center configuration [Fig. 1(a)] is unstable. The reason is the following: $\mathrm{SiAu}_{4}$ is found to be a closed shell with $T_{d}$ symmetry and the bond length of $\mathrm{Au}-\mathrm{Si}$ being $2.299 \AA{ }^{2}$ Although the $T_{d}$ symmetry in the on-center configuration of $\mathrm{Si}-\mathrm{Au}_{16}[\mathrm{Fig}$. 1(a)] can make $\mathrm{Si}$ to be $s p^{3}$-like, the $\mathrm{Au}-\mathrm{Si}$ bond length is $2.51 \AA$, more than $0.2 \AA(\sim 9.2 \%)$ larger than that in $\mathrm{SiAu}_{4}{ }^{2}$ Therefore, the cavity of $\mathrm{Au}_{16}$ cage is too big for encapsulating the $\mathrm{Si}$ atom at the center. Consequently, the off-center configuration is more preferable, similar to that in $\mathrm{La}-\mathrm{C}_{60}$.

To study the stability of the off-center configuration further, we carried out simulated annealing. The simulation lasted for 15 ps with a time step of $1 \mathrm{fs}$. The temperature was gradually reduced from 800 to $0 \mathrm{~K}$ as the simulation proceeded. Finally it was found that the $\mathrm{Si}$ atom emerges from the cage and resides on its surface with one Au atom forming a Au-Si bond, as shown in Fig. 1(c). Starting from the oncenter configuration in Fig. 1(a) and following the same proceesure, we still found the geometry to converge to that of Fig. 1(c), which is 0.617 and $0.457 \mathrm{eV}$ lower in energy than that of on-center and off-center configurations, respectively.
Due to the lower symmetry as compared to the other two structures, the energy gap between highest occupied and lowest unoccupied molecular orbitals (HOMO-LUMO gap) is reduced, but still having a value of $1.46 \mathrm{eV}$ that is comparable to that of $\mathrm{C}_{60}(1.57 \mathrm{eV}) .{ }^{17}$ To further check the stability of the complex in Fig. 1(c), we switched the positions of $\mathrm{Si}$ and the $\mathrm{Au}$ atom it is bonded to, and reoptimized the geometry with the $\mathrm{Si}$ atom inside the cage. However, after simulated annealing, the $\mathrm{Si}$ atom again came out of cage. These simulations clearly indicate that intrinsically Si impurity prefers to reside on the cage surface rather than in its interior.

The HOMO and LUMO are shown in Figs. 2(a) and 2(b). Si atom contributes more to LUMO than to HOMO. Figure 2(c) shows the charge differene isosurface with positive value, defined as the difference between the total density and the isolated atoms. We clearly see that charge accumulates on bonds between $\mathrm{Au}-\mathrm{Au}$ and $\mathrm{Au}-\mathrm{Si}$. Therefore, covalent bonding is dominant in this structure. Similar features are also found for on-center and off-center configurations, as shown in Fig. 3. Inside the cage, $\mathrm{Si}-\mathrm{Au}$ bonding is also found to be more covalentlike. The dominant covalent bonding features make it questionable to apply jellium model to the stability of $\mathrm{Si}-\mathrm{Au}_{16}$, as the jellium model essentially requires metalliclike bonding so that the valence electrons are freelike.

Up to now we have demonstrated that the on-surface geometric configuration is much more stable in energy than that of off-center endrohedral complex. Unfortunately, there are no experimental techniques that can verify the predicted structures directly. It is customary to compare the computed properties of various isomers with experiments and a good

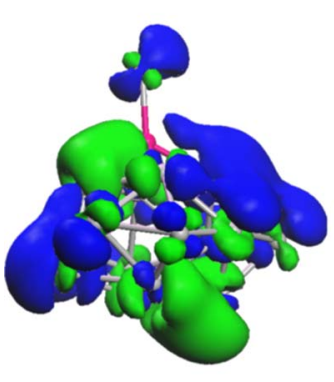

(a)

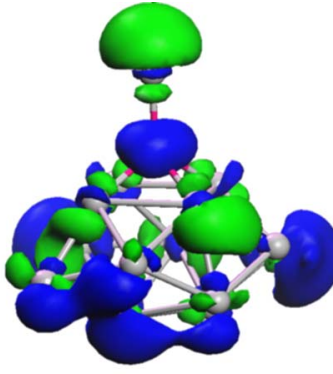

(b)

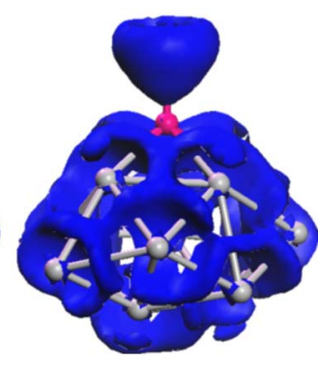

(c)
FIG. 2. (Color online) (a) HOMO and (b) LUMO of $\mathrm{SiAu}_{16}$. (c) The difference charge distribution corresponding to the geometry of Fig. 1(c). 


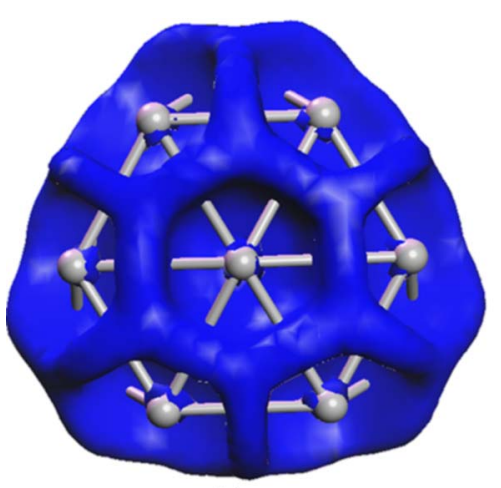

(a)

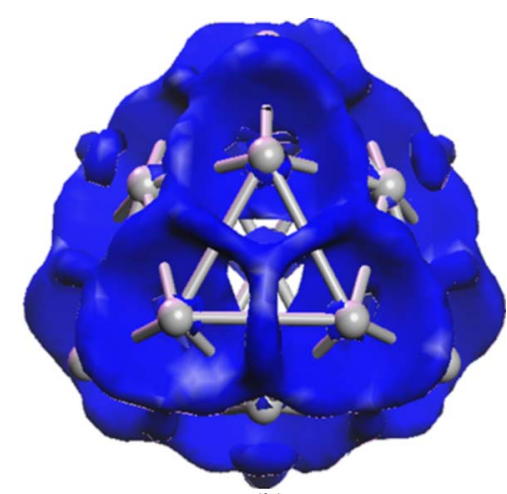

(b)
FIG. 3. (Color online) Difference charge density distribution of $\mathrm{SiAu}_{16}$ corresponding to the geometry of Figs. 1(a) and 1(b), respectively. agreement between theory and experiment serves as an indirect evidence for the predicted geometry. These experiments include photoelectron spectroscopy (PES) as well as infrared (IR) spectroscopy. The PES measures among other features the HOMO-LUMO gap. We find this gap for the two isomers to be 1.72 and $1.46 \mathrm{eV}$, respectively. The difference is rather small and one has to find other characteristic features that can distinguish between the two isomers. We have calculated the frequency and IR intensity of these isomers and find rather large differences. For the off-center geometry, as shown in Fig. 1(b), there are three peaks of IR intensity with values of $2.59,8.49$, and $8.49 \mathrm{~km} / \mathrm{mol}$ located at 220,248 , and $251 \mathrm{~cm}^{-1}$, respectively. These three vibrational modes are mainly contributed by the $\mathrm{Si}$ atom. However, for the on-surface geometry [see Fig. 1(c)], there are only two modes with the IR intensities of 2.26 and $45.85 \mathrm{~km} / \mathrm{mol}$ located at 137 and $453 \mathrm{~cm}^{-1}$. The first mode originates from the $\mathrm{Au}$ atom directly bonding with $\mathrm{Si}$ atom, and the second mode is from the Si atom. The IR intensity of the later is 20 times larger than that of the former. Compared to the offcenter geometry, the IR intensity is five times larger. Therefore, infrared spectroscopy would be an effective means to detect these two isomers: high IR intensity and high frequency corresponding to the on-surface configuration, while low IR intensity and low frequency corresponding to the endohedral configuration.

As we see from above, the off-center configuration is a metastable state. How can then one put a $\mathrm{Si}$ atom inside the cage to form a $\mathrm{Si}-\mathrm{Au}_{16}$ complex? After comprehensive simulation, we came to the conclusion that once $\mathrm{Au}_{16}$ is formed, it is extremely difficult to introduce a Si impurity from outside to the inside of the cage. As Si atom attempts to pass through the cage surface, it always sticks on the surface. So one possible way to form $\mathrm{Si}-\mathrm{Au}_{16}$ is to use $\mathrm{Si}$ impurity as (a)

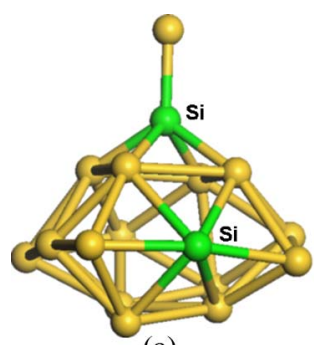

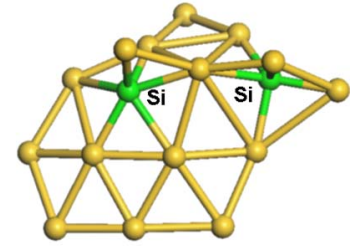

(b)
FIG. 4. (Color online) Initial (a) and final (b) structures of $\mathrm{Si}_{2} \mathrm{Au}_{15}$. a nucleation center, and then introduce $\mathrm{Au}$ atoms. However, the synthesis temperature should not be too high, otherwise due to the much smaller atomic mass of $\mathrm{Si}$ atom, it may diffuse to the surface. In addition, one needs to control the doping concentration of $\mathrm{Si}$ since the cage structure may break if there are too many $\mathrm{Si}$ atoms. We demonstrate this by replacing two $\mathrm{Au}$ atoms in Fig. 1(c) with two $\mathrm{Si}$ atoms to form $\mathrm{Si}_{2} \mathrm{Au}_{15}$ cluster. The optimized geometry is shown in Fig. 4. Note that it has a sheetlike structure where the $\mathrm{Au}$ cage is completely broken. This is in agreement with the fact $^{18}$ that silicon causes fracture and embrittlement in gold jewelry during the manufacturing process where silicon is added to increase the fluidity of molten gold. It is also interesting to note that the two-dimensional-like $\mathrm{Au}-\mathrm{Si}$ structure as shown in Fig. 4(b) is similar to what happens in eutectic liquid surface where a crystalline monolayer is formed. ${ }^{19}$

In summary, using $a b$ initio simulated annealing method we studied the stability of $\mathrm{Si}-\mathrm{Au}_{16}$ and find that the endohedral configuration is metastable. Instead, Si atom prefers bonding on the surface site of gold cluster, similar to what happened between $\mathrm{Si}_{60}$ and $\mathrm{Au}_{12} \mathrm{~W}$ clusters, ${ }^{20}$ sharing some features found in bulk and liquid phases. Doping of $\mathrm{Si}$ in high concentration would cause fracture and embrittlement in gold nanostructure. Our study provides insight on the interactions of $\mathrm{Au}-\mathrm{Si}$ at nanoscale which can be important in the design of new hybrid $\mathrm{Au}-\mathrm{Si}$ nanostructures for applications in microelectronics, catalysis, biomedine, and jewelry industry.

This work is partly supported by Peking University and the Department of Energy.

\footnotetext{
${ }^{1}$ H. Hammer and J. K. Norskov, Nature (London) 376, 238 (1995).

${ }^{2}$ B. Kiran, X. Li, H.-J. Zhai, L.-F. Cui, and L.-S. Wang, Angew. Chem., Int. Ed. 43, 2125 (2004).

${ }^{3}$ M. Haruta, Catal. Today 36, 153 (1997).

${ }^{4}$ P. Pyykko, Angew. Chem., Int. Ed. 43, 4412 (2004).

${ }^{5}$ S. Bulusu, X. Li, L.-S. Wang, and X. C. Zeng, Proc. Natl. Acad. Sci. U.S.A. 103, 8326 (2006).

${ }^{6}$ Q. Sun, P. Jena, Y. D. Kim, M. Fischer, and G. Gantefor, J. Chem. Phys. 120, 6510 (2004).

${ }^{7}$ Q. Sun, B. V. Reddy, M. Marquez, P. Jena, C. Gonzalez, and Q. Wang, J. Phys. Chem. 111, 4159 (2007).

${ }^{8}$ Q. Sun, A. K. Kandalam, Q. Wang, P. Jena, Y. Kawazoe, and M. Marquez, Phys. Rev. B 73, 134409 (2006).

${ }^{9}$ Q. Sun, Q. Wang, B. K. Rao, and P. Jena, Phys. Rev. Lett. 93, 186803 (2004).

${ }^{10}$ M. Walter and H. Hakkinen, Phys. Chem. Chem. Phys. 8, 5407 (2006).

${ }^{11}$ Q. Sun, Q. Wang, J. Z. Yu, V. Kumar, and Y. Kawazoe, Phys. Rev. B 63,
} 
193408 (2001).

${ }^{12}$ G. Kresse and J. Joubert, Phys. Rev. B 59, 1758 (1999).

${ }^{13}$ G. Kresse and J. Furthmüller, Phys. Rev. B 54, 11169 (1996).

${ }^{14} \mathrm{~B}$. Douglas, Concepts and Models of Inorganic Chemistry, 2nd ed. (Wiley, New York, 1983).

${ }^{15}$ J. J. Scherer, J. B. Paul, C. P. Collier, A. O'Keeffe, and R. J. Saykally, J. Chem. Phys. 103, 9187 (1995).

${ }^{16}$ Q. Sun, Q. Wang, P. Jena, R. Note, J.-Z. Yu, and Y. Kawazoe, Phys. Rev. B 70, 245411 (2004).
${ }^{17}$ X. B. Wang, C. F. Ding, and L. S. Wang, J. Chem. Phys. 110, 8217 (1999).

${ }^{18}$ D. Ott, Gold Technology 34, 37 (2002).

${ }^{19}$ O. G. Shpyrko, R. Streitel, V. S. K. Balagurusamy, A. Y. Grigoriev, M. Deutsch, B. M. Ocko, M. Meron, B. Lin, and P. S. Pershan, Science 313, 77 (2006).

${ }^{20}$ Q. Sun, Q. Wang, Y. Kawazoe, and P. Jena, Eur. Phys. J. D 29, 231 (2004). 